\title{
Estudio genético multiloci en la población colombiana. II. Distribución del complejo CcDdEe del grupo sanguíneo $\mathbf{R h}$
}

\author{
Jorge A. Díaz', Indiana Bustos ${ }^{2}$
}

\begin{abstract}
Resumen
La población colombiana se caractersizó para el complejo CcDdEe del grupo sanguíneo $\mathrm{Rh}$; se estudiaron diez regiones para dos generaciones; las regiones mostraron ser genéticamente homogéneas para el factor $\mathrm{Rh}$, mientras que para el complejo CcDdEe y para los sistemas codominantes $\mathrm{Cc}$ y Ee fueron heterogéneas. La influencia de las diferentes razas en la población colombiana fue detectada a través de los fenotipos CCD-EE, CcD-Ee, CCddee, Ccddee, ccD-ee, ccddEe y ccddee.
\end{abstract}

\section{Summary}

For two generations, the Rh blood group was studied in ten different Colombian regions to characterise the Colombian population for the CcDdEe complex in the $\mathrm{Rh}$ blood group. These regions proved to be genetically homogenous for the $\mathrm{Rh}$ factor, whereas the CcDdEe complex and co-dominant Cc and Ee systems were shown to be heterogenous. The preponderance of different races in the Colombian population was thus detected by using CCD-EE, CcD-Ee, CCddee, Ccddee, ccD-ee, ccddEe and ccddee phenotypes.

Los grupos sanguíneos han sido usados frecuentemente en problemas de parentezco, dentro de los cuales se incluyen: exclusión de la maternidad, por intercambio de recién nacidos, disputas de paternidad y ubicación de individuos dentro de grupos raciales, cuando existen marcadores genéticos.

Dentro de los grupos sanguíneos, el factor $\mathrm{Rh}$ (Dd) es de gran importancia por la causalidad de reacciones hemolíticas y por la muerte hemolítica del recién nacido, además de ser un marcador genético importante.

El grupo sanguíneo Rh está determinado por tres loci, para los que se reconocen cerca de

1 Biólogo, Universidad Nacional; investigador independiente.

2 Profesora asociada, Universidad Nacional; directora, Departamento de Biología.
25 antígenos, de los cuales son de uso general por sus altas frecuencias en las diferentes poblaciones los antígenos: anti-C, anti-c, anti-E, anti-e y anti-D (1). El complejo de genes del sistema $R$ h se hereda en forma simple y los individuos se pueden analizar en términos del conjunto o de los sistemas individuales, presentando los sistemas Cc y Ee codominancia y el sistema Dd dominancia completa $(2,3)$.

En un pequeño grupo de indígenas llamado Ica, localizado en la Sierra Nevada de Santa Marta, Colombia, se encontró que cerca del $20 \%$ de los complejos de genes del grupo sanguíneo $\mathrm{Rh}$ eran de un nuevo género, $\mathrm{cDe}$. Las características principales de este nuevo factor $\mathrm{Rh}$ son que reacciona con pequeñas cantidades de suero anti-e y que el cromosoma que lleva $e^{i}$ tiene $D$, c y f (4). 


\section{Materiales y métodos}

En este estudio se caracterizó la población colombiana para el complejo CcDdEe del grupo sanguíneo $\mathrm{Rh}$ y para los sistemas indepen- dientes para diez regiones geográficas del país en dos generaciones: padres e hijos (figura 1).

Las frecuencias génicas y fenotípicas observadas y esperadas para los sistemas codo-

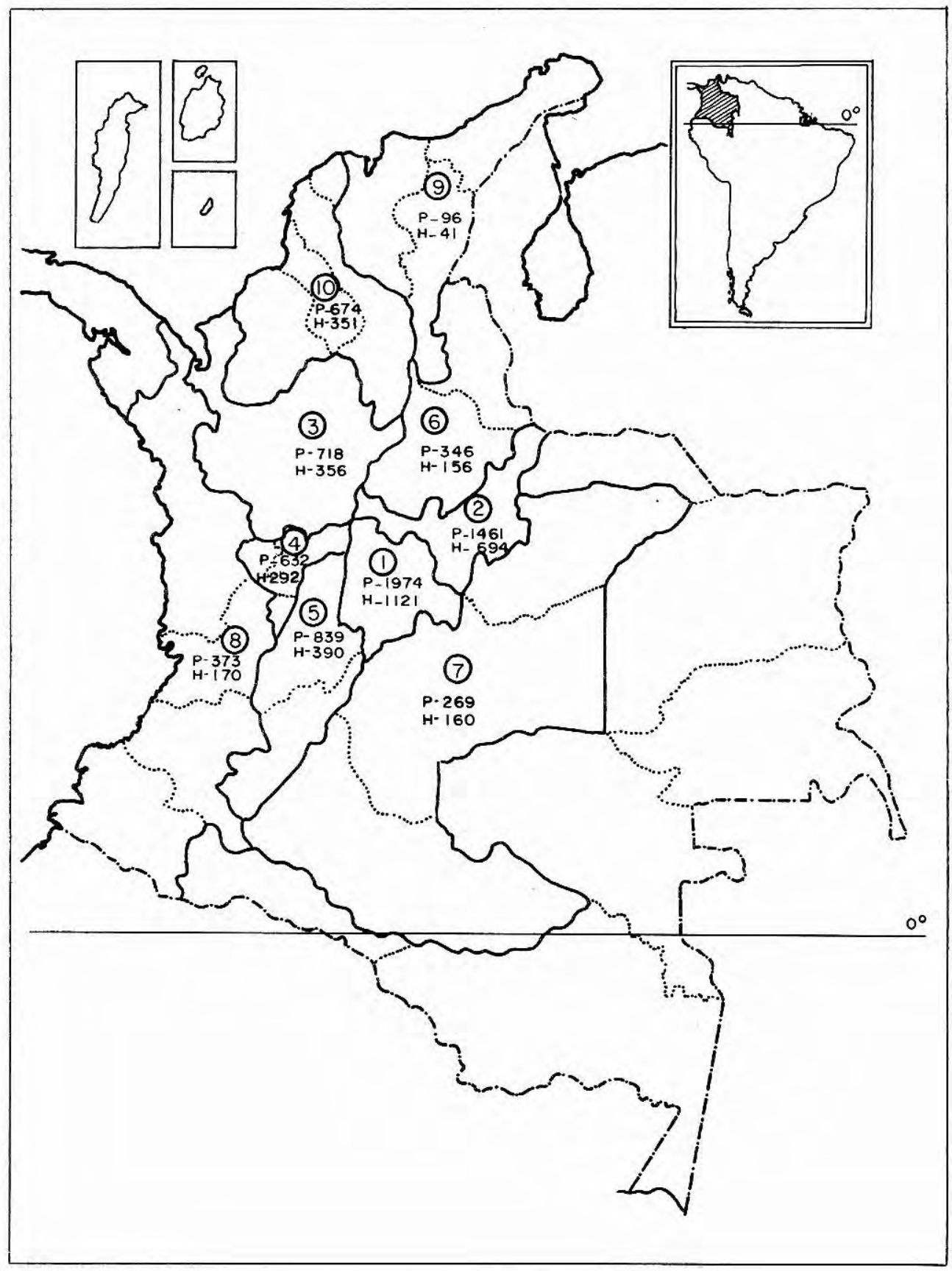

Figura 1. Número de individuos estudiados por regiones y generaciones. 
minantes (Cc y Ee), para el sistema dominante (factor Rh) y para el complejo CcD-Ee se calcularon por la metodología clásica (5).

Para determinar el ajuste al equilibrio de HardyWeinberg, se utilizó la prueba estadística chi cuadrado $\mathrm{T}\left(\mathrm{X}_{\mathrm{T}}{ }_{\mathrm{T}}\right)$ y para establecer la homogeneidad entre las frecuencias génicas $y$ fenotípicas de las regiones y las generaciones se utilizó la prueba de chi cuadrado de homogeneidad, $X_{H}^{2}(6)$. Para las frecuencias génicas se empleó la ecuación de Brandt y Snedecor (7).

La metología y descripción de la población empleada en este trabajo puede ser vista en detalle en el trabajo de Díaz, 1988 (8).

\section{Resultados}

El orden encontrado para las frecuencias fenotípicas del sistema $\mathrm{Cc}$ en las regiones para las dos generaciones es: $\mathrm{Cc}>\mathrm{cc}>\mathrm{CC}$, a excepción de las regiones 5 (Tolima y Huila) y 7 (Meta, Caquetá y Casanare) en donde la frecuencia del fenotipo $\mathrm{CC}$ es mayor que la frecuencia del fenotipo cc (figuras 2 y 3 ).

En la generación paterna, todas las regiones y la población total se encuentran en equilibrio de Hardy-Weinberg (tabla 1), en tanto que en la generación filial las regiones 4 (Caldas, Quindío y Risaralda), 9 (La Guajira, Cesar y Magdalena) y 10 (Atlántico, Bolívar, Córdova, Sucre y San Andrés) se encuentran en desequilibrio. En la región 9 se encontró además que las frecuencias fenotípicas entre padres e hijos son heterogéneas, mientras que en las regiones 4 y 10 las dos generaciones son homogéneas y en la región 7 aunque las dos generaciones se encuentran en equilibrio, las frecuencias fenotípicas de padres e hijos mostraron ser heterogéneas entre sí. La distribución de frecuencias para el sistema $\mathrm{Cc}$ entre 8 las regiones dentro de cada generación mostraron ser altamente heterogéneas $\left(\mathrm{X}_{\mathrm{Hp}}^{2}=68,18\right.$ y $\mathrm{X}_{\mathrm{Hh}}^{2}=$ 61,45).

Para el sistema Ee, el fenotipo ee fue el más frecuente, seguido por el fenotipo heterocigoto; este comportamiento de las frecuencias fenotípicas se encontró en todas las regiones y en

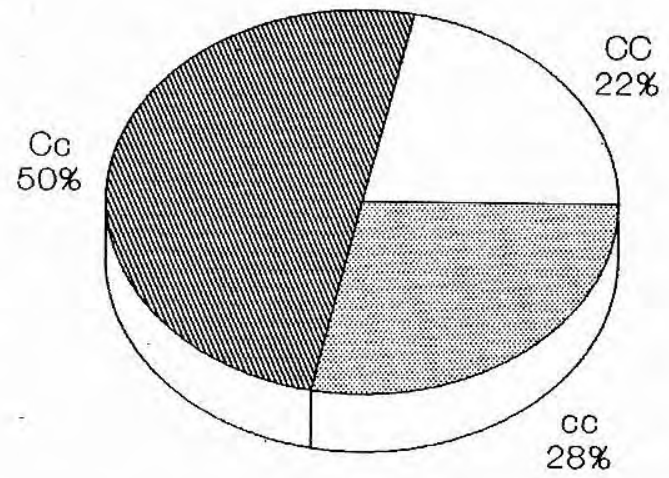

Figura 2. Distribución fenotípica para el sistema Cc en la población colombiana.

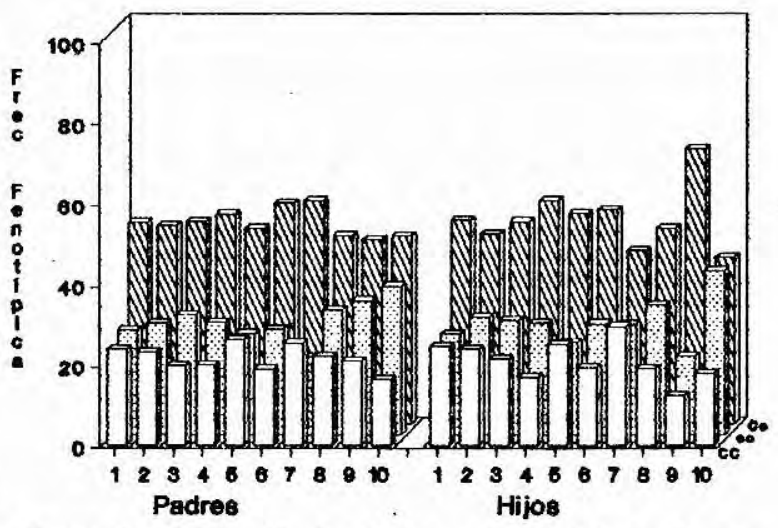

Figura 3. Distribución fenotípica para el sistema Cc por regiones y generaciones en la población colombiana.

la población total para las dos generaciones (figuras 4 y 5). En todos los casos se presentó equilibrio de Hardy y Weinberg y las frecuencias fenotípicas de los padres fueron homogéneas con las de los hijos en todas las regiones y en la población total (tabla 1). Sin embargo, existe heterogeneidad entre las regiones dentro de cada generación $\left(X^{2}{ }_{H p}=99,26\right.$ y $\left.X^{2}{ }_{H h}=59,79\right)$. La región 2 presentó las menores frecuencias para el fenotipo ee, así como las frecuencias más altas para el fenotipo EE.

Las frecuencias fenotípicas para el sistema Dd del grupo sanguíneo $\mathrm{Rh}$ (figuras 6 y 7 ) mostraron un comportamiento uniforme en las diferentes regiones y en la población total para 


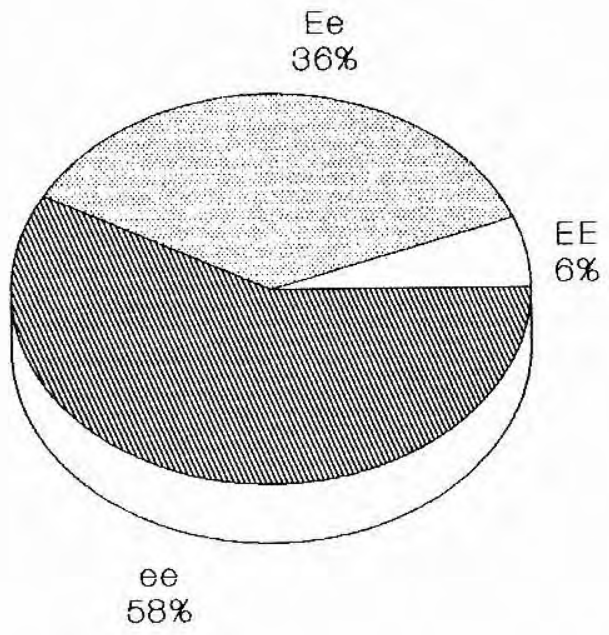

Figura 4. Distribución fenotípica para el sistema Ee en la población colombiana.

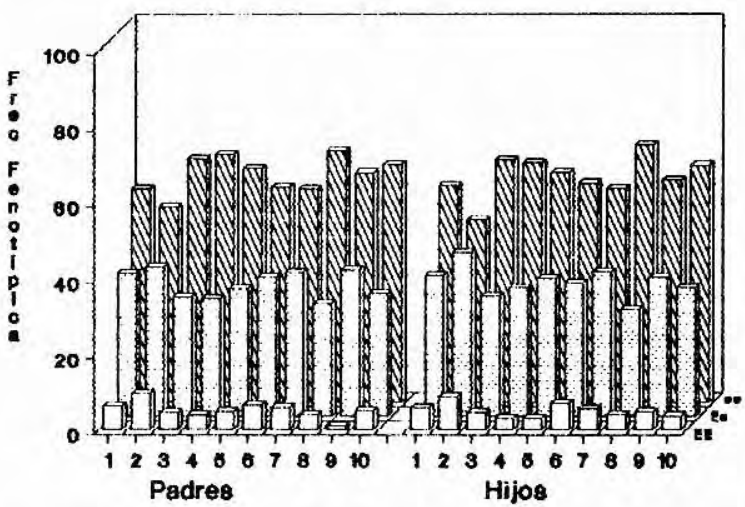

Figura 5. Distribución fenotípica para el sistema Ee por regiones y generaciones en la población colombiana.

las dos generaciones. Las frecuencias fenotípicas más altas para el $\mathrm{Rh}+\mathrm{se}$ encontraron en la región 9 para la generación de los hijos y en la región 7 para la generación de los padres $(0,9756$ y 0,9650$)$, en tanto que la región 4 presentó los valores más altos para el fenotipo Rh-: 0,0820 para padres y 0,0769 para hijos (figura 7).

El orden de la distribución fenotípica presentado por la población colombiana total para el
Tabla 1. Valores de $\mathrm{XT}^{2}$ y $\mathrm{XH}^{2}$ para los sistemas codominantes por regiones y generaciones.

\begin{tabular}{|c|c|c|c|c|c|c|}
\hline \multirow[b]{2}{*}{ Región } & \multicolumn{3}{|c|}{ Cc } & \multicolumn{3}{|c|}{ Ee } \\
\hline & $\mathrm{X}_{\mathrm{TP}}$ & $\mathbf{X}^{2} \mathrm{H}$ & $\mathbf{X}^{\mathbf{Z}_{\text {Th }}}$ & $\mathrm{X}_{\mathrm{TP}}$ & $\mathbf{X}^{\mathbf{Z}_{\mathrm{H}}}$ & $\mathrm{X}_{\mathrm{Th}}$ \\
\hline 1 & 0,060 & 0,370 & 0,202 & 0,027 & 0,520 & 0,130 \\
\hline 2 & 0,176 & 0,874 & 1,870 & 3,321 & 2,820 & 0,260 \\
\hline 3 & 0,264 & 0,546 & 0,040 & 0,660 & 0,020 & 0,160 \\
\hline 4 & 1,626 & 1,451 & $4,423^{*}$ & 0,100 & 1,150 & 0,990 \\
\hline 5 & 0,522 & 1,424 & 0,847 & 0,038 & 2,460 & 2,930 \\
\hline 6 & 3,779 & 0,133 & 0,799 & 0,160 & 0,108 & 0,420 \\
\hline 7 & 3,359 & 6,590 * & 3,012 & 0,050 & 0,019 & 0,100 \\
\hline 8 & 1,080 & 0,703 & 0,005 & 0,320 & 0,130 & 0,480 \\
\hline 9 & 0,454 & $5,812^{*}$ & 5,711 * & 3,490 & 1,980 & 0,030 \\
\hline 10 & 0,381 & 2,474 & $5,164^{*}$ & 0,630 & 1,040 & 0,410 \\
\hline Total & 0,030 & 0,131 & 0,098 & 3,720 & 2,706 & 0,440 \\
\hline
\end{tabular}

complejo CcDdEe, solamente se encontró en la región 6 (Santander y Norte de Santander) en los padres y los hijos: en la región 1 (Cundinamarca) sólo en los padres y en la región 4 sólo en los hijos; el resto de las regiones muestra distribuciones fenotípicas diferentes.

Se encontraron tres grupos de frecuencias fenotípicas en la población colombiana: el primero formado por los fenotipos CCD-ee, $\mathrm{CcD}-\mathrm{Ee}$ y $\mathrm{CcD}$-ee con frecuencias mayores del $20 \%$; los fenotipos ccD-EE, ccD-Ee, ccD-ee y ccddee con frecuencias entre el 4 y $13,5 \%$ y los demás fenotipos del complejo con frecuencias inferiores al $1 \%$ (tablas 2 y 3 ).

La distribución de las frecuencias fenotípicas para el complejo CcDdEe entre padres e hijos mostró las siguientes diferencias: en los hijos no se encontró el fenotipo CCD-EE, pero sí está presente el fenotipo CCddee. De otro lado, la frecuencia del fenotipo Ccddee es mayor que la frecuencia del fenotipo CCD-Ee, contrario a lo encontrado en la generación paterna. Los fenotipos CCddEE, CCddEe, CcddEE, CcddEe y ccddEE no se encontraron en la población colombiana en ninguna de las generaciones. El fenotipo CCD-EE, solamente se encontró en la región 7 (Meta, Caquetá y Casanare) en la generación paterna (tablas 2 y 3 ). 


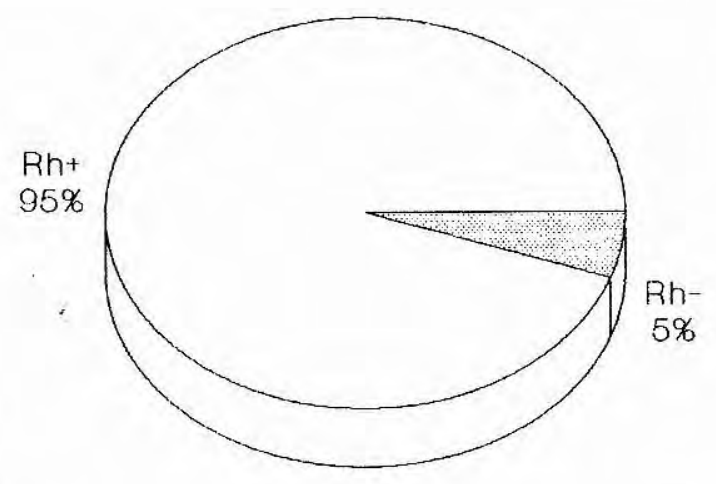

Figura 6. Distribución fenotípica para el grupo sanguíneo Rh en la población colombiana.

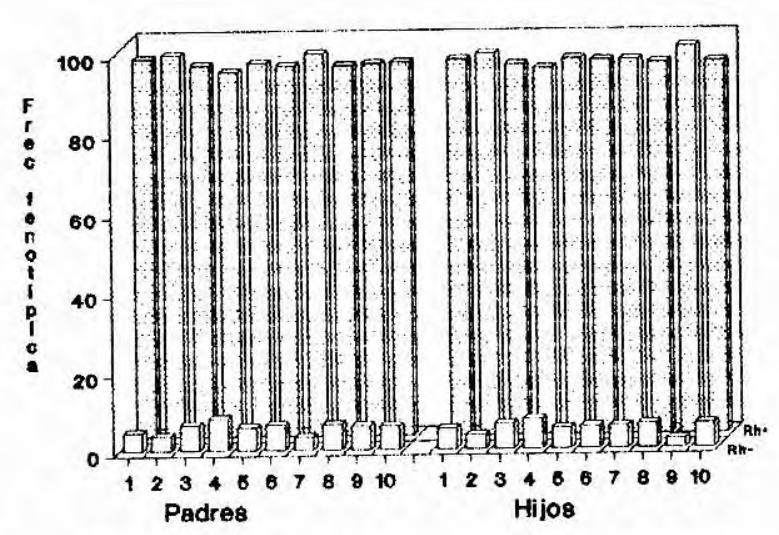

Figura 7. Distribución fenotípica para el grupo sanguíneo $\mathrm{Rh}$ por regiones y generaciones en la población colombiana.

Tabla 2. Frecuencias fenotípicas para el complejo CcD-Ee del grupo sanguíneo Rh para la generación paterna en la población colombiana.

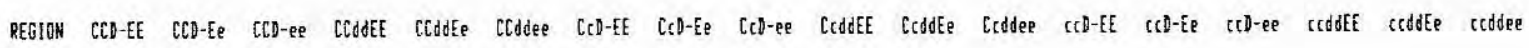

$\begin{array}{lllllllllllllllllllllll}1 & 0.0000 & 0.0046 & 0.2335 & 0.0000 & 0.0000 & 0.0000 & 0.0010 & 0.2421 & 0.2584 & 0.0000 & 0.0000 & 0.0010 & 0.0613 & 0.1297 & 0.0213 & 0.0000 & 0.0010 & 0.0461 \\ 2 & 0.0000 & 0.0027 & 0.2279 & 0.0000 & 0.0000 & 0.0000 & 0.0014 & 0.2635 & 0.2279 & 0.0000 & 0.0000 & 0.0007 & 0.0931 & 0.1259 & 0.0198 & 0.0000 & 0.0007 & 0.0363 \\ 3 & 0.0000 & 0.0028 & 0.1936 & 0.0000 & 0.0000 & 0.0000 & 0.0000 & 0.1825 & 0.3189 & 0.0000 & 0.0000 & 0.0028 & 0.0460 & 0.1212 & 0.0710 & 0.0000 & 0.0070 & 0.0543 \\ 4 & 0.0000 & 0.0000 & 0.1994 & 0.0000 & 0.0000 & 0.0000 & 0.0016 & 0.1867 & 0.3323 & 0.0000 & 0.0000 & 0.0016 & 0.0380 & 0.1203 & 0.0396 & 0.0000 & 0.0016 & 0.0791 \\ 5 & 0.0000 & 0.0000 & 0.2613 & 0.0000 & 0.0000 & 0.0000 & 0.0000 & 0.2160 & 0.2673 & 0.0000 & 0.0000 & 0.0048 & 0.0477 & 0.1193 & 0.0298 & 0.0000 & 0.0012 & 0.0525 \\ 6 & 0.0000 & 0.0029 & 0.1850 & 0.0000 & 0.0000 & 0.0000 & 0.0000 & 0.2370 & 0.3092 & 0.0000 & 0.0000 & 0.0029 & 0.0665 & 0.1243 & 0.0116 & 0.0000 & 0.0029 & 0.0578 \\ 7 & 0.0037 & 0.0000 & 0.2500 & 0.0000 & 0.0000 & 0.0000 & 0.0037 & 0.2836 & 0.2649 & 0.0000 & 0.0000 & 0.0000 & 0.0485 & 0.0970 & 0.0112 & 0.0000 & 0.0000 & 0.0373 \\ 8 & 0.0000 & 0.0027 & 0.2172 & 0.0000 & 0.0000 & 0.0000 & 0.0027 & 0.1850 & 0.2735 & 0.0000 & 0.0000 & 0.0080 & 0.0375 & 0.1099 & 0.1019 & 0.0000 & 0.0000 & 0.0617 \\ 9 & 0.0000 & 0.0208 & 0.1875 & 0.0000 & 0.0000 & 0.0000 & 0.0000 & 0.1875 & 0.2604 & 0.0000 & 0.0000 & 0.0104 & 0.0104 & 0.1771 & 0.0938 & 0.0000 & 0.0000 & 0.0521 \\ 10 & 0.0000 & 0.0074 & 0.1558 & 0.0000 & 0.0000 & 0.0000 & 0.0030 & 0.1558 & 0.3027 & 0.0000 & 0.0000 & 0.0059 & 0.0475 & 0.1617 & 0.1024 & 0.0000 & 0.0000 & 0.0579\end{array}$

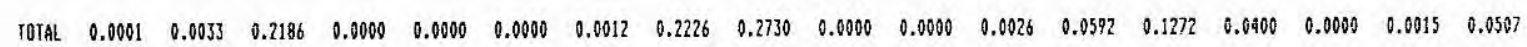


Tabla 3. Frecuencias fenotípicas para el complejo $\mathrm{CcD}$-Ee del grupo sanguíneo Rh para la generación filial en la población colombiana.

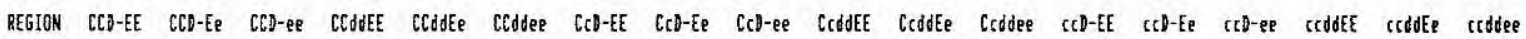

\begin{tabular}{|c|c|c|c|c|c|c|c|c|c|c|c|c|c|c|c|c|c|c|}
\hline 1 & 0.0000 & 0.0009 & 0.2426 & 0.0000 & 0.0000 & 0.0000 & 0.0018 & 0.2409 & 0.2614 & 0.0000 & 0.0000 & 0.0027 & 0.0553 & 0.1285 & 0.0196 & 0.0000 & 0.0018 & 0.0446 \\
\hline 2 & 0.0000 & 0.0043 & 0.2320 & 0.0000 & 0.0000 & 0.0000 & 0.0014 & 0.2810 & 0.1902 & 0.0000 & 8.0000 & 0.0000 & 0.0865 & 0.1441 & 0.0216 & 0,0000 & 0.0014 & 0.0375 \\
\hline 3 & 0.0000 & 0.0000 & 0.2135 & 0.0000 & 0.0000 & 0.0000 & 0.0000 & 0.2022 & 0.2949 & 0.0000 & 0.0000 & 0.0056 & 0.0449 & 0.1096 & 0.0618 & 0.0000 & 0.0056 & 0.0618 \\
\hline 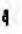 & 0.0000 & 0.0000 & 0.1678 & 0.0000 & 0.0000 & 0.0800 & 0.0034 & 0.2158 & 0.3322 & 0.0000 & 0.0000 & 0.0034 & 0.0274 & 0.1233 & 0.0514 & 0.0000 & 0.0000 & 0.0753 \\
\hline 5 & 0.0000 & 0.0026 & 0.2462 & 0.0000 & 0.0000 & 0.0000 & 0.0000 & 0.2359 & 0.2821 & 0.0000 & 0.0000 & 0.0051 & 0.0308 & 0.1256 & 0.0179 & 0.0000 & 0.0000 & 0.0538 \\
\hline 6 & 0.0000 & 0.0000 & 0.1923 & 0.0000 & 0.0000 & 0.0000 & 0.0000 & 0.2372 & 0.2949 & 0.0000 & 0.0000 & 0.0000 & 0.0705 & 0.1154 & 0.0381 & 0.0000 & 0.0000 & 0.0513 \\
\hline 7 & 0.0000 & 0.0125 & 0.2813 & 0.0000 & 0.0000 & 0.0000 & 0.0000 & 0.2313 & 0.2000 & 0.0000 & 0.0000 & 0.0000 & 0.0563 & 0.1313 & 0.0250 & 0.0000 & 0.0063 & 0.0563 \\
\hline 8 & 0.0000 & 0.0059 & 0.1824 & 0.0000 & 0.0000 & 0.0000 & 0.0000 & 0.1529 & 0.3294 & 0.0000 & 0.0000 & 0.0059 & 0.0412 & 0.1235 & 0.1000 & 0.0000 & 0.0000 & 0.0588 \\
\hline 4 & 0.0000 & 0.0244 & 0.0876 & 0.0000 & 0.0000 & 0.0000 & 0.0000 & 0.21995 & 0.4390 & 0.0000 & 0.0000 & 0.0244 & 0.0486 & 0.1220 & 0.2440 & 0.0000 & 0.0000 & 0.0000 \\
\hline & 0.0000 & 0.0028 & 0.1709 & 0.0000 & 0.0000 & 0.0028 & 0.0028 & 0.1481 & 0.2621 & 0.0000 & 0.0000 & 0.0028 & 0.0342 & 0.1880 & 0.1225 & 0.0000 & 0.0000 & 1627 \\
\hline
\end{tabular}

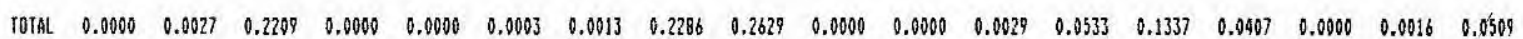

\section{Discusión}

La presencia de desequilibrio en las regiones 4 , 9 y 10 , puede ser atribuida al efecto de la selección que favorece al fenotipo $\mathrm{Cc}$ en las dos primeras regiones y a los fenotipos homocigotos en la región 10; es importante señalar que las regiones 9 y 10 comprenden departamentos de una misma región natural (costa atlántica) y, por tanto, a grupos étnicos muy similares sobre los cuales posiblemente deberían operan condiciones ambientales semejantes. Sin embargo, los resultados indican un efecto contradictorio, lo cual puede ser explicado en parte por el tamaño reducido de la muestra de la región 9 .

La presencia del fenotipo Rh- en la población colombiana, es atribuida al aporte de las razas blanca y negra, ya que este fenotipo no existía en las poblaciones indígenas colombianas (4).

El orden de las frecuencias fenotípicas hallado en este trabajo coincide en gran parte con el encontrado por Gómez en 1981, quien trabajó con 3.273 individuos de la población colombiana (9). Es importante anotar que el fenotipo CCD-EE presente en la generación paterna y el fenotipo CCddee presente en la generación filial, no fueron encontrados por Gómez; estas diferencias pueden ser atribuidas al tamaño mayor de la muestra usada en este trabajo.

La existencia del fenotipo ccddee en la población colombiana con una frecuencia cercana al $5 \%$, puede ser atribuida a la mezcla de la población indígena con la raza blanca y en menor proporción a la mezcla con la raza negra, 
teniendo en cuenta que la gameta cde no se ha encontrado en poblaciones indígenas y en los casos en que se ha encontrado su presencia es atribuida a la mezcla con individuos de la raza blanca y de la raza negra $(4,10,11)$, para las cuales se señalan valores de 38,4 y $11,84 \%$ respectivamente (5). De otro lado, la región 4 (Caldas, Quindío y Risaralda) presentó la frecuencia más alta para el fenotipo ccddee, mostrando así la mayor incidencia de la raza blanca en esta región, si bien se tiene en cuenta que en algunas poblaciones españolas la gameta cde alcanza valores hasta del $53,13 \%(12)$.

Adicionalmente, estudios realizados en poblaciones españolas indican que además de los fenotipos ausentes en la población colombiana, no se encontraron los fenotipos CCD-EE, CCDee y CcD-EE en Galicia y en Bascos, en Bascos. Además, no se encontró el fenotipo ccDEE y es notable la alta frecuencia de los fenotipos CcD-ee $(0,4204-0,4321)$ y ccddee $(0,2742-0,2222)(12-14)$.

En un estudio realizado con una muestra de 8.297 niños suecos, no se encontraron los fenotipos ausentes en la población colombiana ni los fenotipos CCD-EE y CCD-ee. De otra parte, las frecuencias fenotípicas encontradas son diferentes a las de la población colombiana; cabe resaltar las altas frecuencias de los fenotipos CcD-Ee $(0,34193)$ y ccddee $(0,14897)$ en la población sueca (15).

La mayor frecuencia del fenotipo Ccddee en las regiones 8 (Chocó, Valle, Cauca y Nariño), 9 (Guajira, Cesar y Magdalena) y 10 (Atlántico, Bolívar, Córdova, Sucre y San Andrés) de las costas colombianas, puede ser atribuida a la presencia de la raza negra en estos departamentos, raza en la que la gameta Cde tiene una frecuencia de 0,0707 (5).

La mayor incidencia de la raza negra en las regiones costeras de Colombia, puede ser detectada más claramente a través del fenotipo ccD-ee, ya que en la raza negra la gameta $\mathrm{cDe}$ tiene una frecuencia de 0,7395 (5), hecho que también es notable en el departamento de
Antioquia (región 3) en donde se encuentran algunas zonas de población negra.

Así mismo, las frecuencias bajas del fenotipo Ccddee en las regiones 1 (Cundinamarca), 2 (Boyacá) y 7 (Meta, Caquetá y Casanare) deja ver la mayor incidencia indígena, ya que la gameta Cde no se ha encontrado en las tribus indígenas sudamericanas $(4,10,11,16)$.

La presencia del fenotipo CCD-EE en la región 7 , no es un hecho sorprendente si se tiene en cuenta que la mayoría de las tribus indígenas sudamericanas localizadas al oriente de la cordillera de los Andes (Cofan, Shiona y Warrua en Venezuela y Macushi, Wapishina y otras en Brasil) presentan una frecuencia relativamente alta de la gameta $\operatorname{CDE}(2 \%-13 \%)$, mientras que en las tribus localizadas sobre la cordillera de los Andes y al occidente de ésta, no se ha encontrado esta gameta $(4,10,11,16)$.

El fenotipo CCD-EE también fue encontrado en la población de aborígenes australianos con una frecuencia de 0,0125 (17) y en la raza mongoloide la gameta CDE presenta una frecuencia de $0,0049(5)$, frecuencia similar a la encontrada en la población colombiana $(0,0046)(9)$.

La ausencia de los fenotipos CCD-EE, CCDEe, CCddEe, CcddEE y CcddEe en la mayoría de las regiones y, por ende, las bajas frecuencias en la población colombiana, puede ser atribuida a la ausencia de la gameta $\mathrm{CdE}$ en las poblaciones indígenas colombianas $(4,10,11$, 16) y a la ausencia de la gameta CDE en las diferentes razas que confluyeron en el país, a excepción de las poblaciones indígenas situadas al occidente de los Andes.

La baja frecuencia de la gameta CE en la población colombiana, puede ser debida a la acción de la selección sobre esta gameta en algunas regiones del país, la cual podría ser realizada por factores selectivos acumulativos sobre los cigotos o individuos que presenten mayor número de alelos $\mathrm{C}$ y $\mathrm{E}$. Además, las frecuencias de los fenotipos formados por estas gametas se mantendrán bajas en la población colombiana debido al ligamiento íntimo pre- 
sente entre los loci del sistema sanguíneo Rh (18).

\section{Conclusiones}

La población colombiana presenta homogeneidad genética para el factor $\mathrm{Rh}(\mathrm{Dd})$, en tanto que para los sistemas codominantes (Cc y Ee) y para el complejo $\mathrm{CcDdEe}$, se encuentra dividida en regiones genéticamente heterogéneas.

La población colombiana, por ser el producto de la mezcla de varias razas (negroide, caucasoide e indígena), presenta una mayor variedad fenotípica para el complejo CcDdEe.

La presencia de los fenotipos CCD-EE y CcDEE en la población colombiana indudablemente se debe al aporte de la raza indígena ya que estos fenotipos se encuentran ausentes en otras poblaciones, en tanto que la existencia de los fenotipos CCddee y Ccddee y las frecuencias altas del fenotipo ccD-ee seguramente son el resultado del aporte de la raza negra, mientras que la presencia del fenotipo ccddEe muestra la incidencia de la raza blanca, ya que la gameta cEd no ha sido encontrada en otras poblaciones.

La incidencia de la raza indígena en las regiones que incluyen los departamentos de Nariño, Cauca y La Guajira no pudo ser establecida en este trabajo debido posiblemente a los pequeños tamaños de la muestra disponible o a los patrones culturales de las etnias indígenas presentes en estas áreas del país.

\section{Referencias}

1. Race R, Sanger R. Blood groups in man, 1968.

2. Wiener AS. Genetic theory of the Rh blood types. Proc Exp Biol NY 1943;54:316-319.

3. Race R, et al. The Rh genotypes and Fisher's theory. Blood 1948;3(2 Suppl):27-42.
4. Layrisse M, Layrisse Z. Blood groups antigen studies of four Chibchan tribes. Amer Antrhopol 1963;65:36-55.

5. Cavalli-Sforza B. Human genetics populations. Freeman WH, 1971.

6. Sokal R. Biometría: principios y métodos básicos en la investigación biológica. H Blume Ediciones, 1979.

7. Kenpthorne $\mathbf{0}$. An introduction to genetics statistics. New York: John Willey and Sons, 1957.

8. Diaz JA. Desequilibrio de ligamiento para el cComplejo CcEe del grupo sanguíneo $\mathrm{Rh}$ en población colombiana (tesis). Bogotá: Universidad Nacional de Colombia, 1988.

9. Gómez A. Estudio de los grupos sanguíneos en la población colombiana y su aplicación en problemas de paternidad (tesis). Bogotá: Universidad Nacional de Colombia, 1981.

10. Layrisse M, Arends T. High incidence blood groups found in Venezuelan indians. Science 1956;123:633.

11. Kirk RL, MacDermi EM, Blacke, Gajducek DC, Leyshin WC, Maclennan R. Blood group serum protein and red cell in enzyme groups of Amerindian populations in Colombia. Amer J Phys Antrhop 1974;41:301315.

12. Chalmers JM, Ikin E, Mourant AE. The ABO, MNS and $\mathrm{Rh}$ blood groups of the Basques people. Amer J Phys Anthrop 1947:529-544.

13. Agostini LR, Ikin E, Mourant AE. Les groupes sanguins $A B O, M N S$ et $R$ h des galiciens (Espagne NO). Rev Hemat 1950;6:325-328.

14. Eyquem MA. Repartition des groupes sanguins chez les Basques. Bul Acad Nat Med 1950;171:7-18.

15. Heiken A, Rasmuson M. Genetical studies on the Rh blood groups system. Heredites Lund 1966;55:192-212.

16. Salzano FM. The blood groups of South American indians. Amer J Phys Antrhop 1957;15:555-579.

17. Cadien JD, Hassett MJ, Friedlaender JF. Linkage desequilibrium in Bougainville island. Amer $\mathrm{J}$ Phys Antrop 1978;48:297-304.

18. Díaz JA, Bustos I. Estudio genético multiloci en la población colombiana. I. Desequilibrio de ligamiento para el sistema CcEe. Biomédica 1994;14:63-68. 